\title{
Exclusión social de la infancia afrocolombiana en el aula escolar desde un enfoque bioético*
}

Social Exclusion of Afro-Colombian Children in the School Classroom from a Bioethical Approach

Exclusão social da infância afro-colombiana na sala de aula escolar desde uma abordagem bioética

Julieth Milena Rincón Perdomo** (iD orcid.org/0000-0002-7665-2705

Artículo de reflexión

Revista Colombiana de Educación, N.․ 76. Primer semestre de 2019, Bogotá, Colombia.

doi: 10.17227/rce.num76-9384

Para citar este artículo: Rincón, J. (2019). Exclusión social de la infancia afrocolombiana en el aula escolar desde un enfoque bioético. Revista Colombiana de Educación, 76, 305-320.

\section{(c) $\underset{\mathrm{BY}}{\mathrm{NC}}$}

Este artículo es parte del proceso de formación doctoral. Doctorado en Bioética de la Universidad El Bosque, Bogotá, Colombia.

** Magíster en Educación. Docente de la Corporación Universitaria Minuto de Dios, Bogotá, Colombia. Correo electrónico: jrinconperd@uniminuto.edu.co 


\section{Resumen}

Este artículo tiene como propósito reflexionar sobre la exclusión social, planteando el problema desde un caso real de un niño afrocolombiano vinculado al aula escolar en el nivel de básica primaria. Si bien existen estudios sobre este fenómeno, es conveniente afianzar los procesos de investigación y reflexión sobre las manifestaciones y las consecuencias que se pueden dar ante este caso. En un segundo momento, se demuestra que el fenómeno de exclusión social, en la población afrocolombiana, ha transcendido de generación en generación a través de la historia. Por último, se analiza el problema desde la bioética, para llegar a su análisis desde los principios éticos básicos del Informe Belmont, que son justicia y respeto por las personas.

\section{Palabras clave}

infancia; discriminación social; justicia social
Keywords

childhood; social discrimination; social justice

\begin{abstract}
This paper aims to reflect on social exclusion, presenting the problem from a real case of an Afro-Colombian child in an elementary school classroom. Although there are studies about this phenomenon, it is important to consolidate the processes of research and reflection on the possible manifestations and consequences of this case. In the second section, the paper demonstrates that the phenomenon of social exclusion in the Afro-Colombian community has transcended from generation to generation throughout history. Finally, the paper analyzes the issue from the perspective of bioethics, in order to reach its analysis from the basic ethical principles of the Belmont Report, namely justice and respect for people.
\end{abstract}

\section{Resumo}

Este artigo visa refletir sobre a exclusão social, colocando o problema desde um caso real de uma criança afro-colombiana vinculada à sala de aula escolar no Ensino Fundamental. Bem se existem estudos sobre esse fenômeno, convém consolidar os processos de pesquisa e reflexão sobre as manifestações e as consequências possíveis nesse caso. Em seguida, demonstramos que o fenômeno de exclusão social, na população afro-colombiana, tem transcendido de geração em geração através da história. Finalmente, analisamos o problema desde a bioética, levando em conta os princípios éticos básicos do Informe Belmont, que são justiça e respeito pelas pessoas.

\section{Palavras-chave}

infância; discriminação social; justiça social 
La escuela es un entorno privilegiado para el estudio del aprendizaje de la relevancia social, de diferencias culturales, donde se puede generar exclusión social de niños y niñas "afrocolombianos(as)" (Castillo, 2009). Las instituciones educativas desempeñan un papel importante en la reproducción de las desigualdades. No obstante, al mismo tiempo incluye grandes posibilidades de transformación de estas (Buile, 2007). El presente escrito constituye un aporte teórico aún en construcción, que pretende plantear una reflexión sobre algunos aspectos fundamentales en el abordaje de la exclusión social de niños y niñas afrodescendientes vinculados al aula escolar.

En la escuela primaria colombiana, la presencia de afrocolombianos se encuentra asociada a estereotipos relacionados con la construcción de una geografía racializada del país, su categorización social y la reproducción de las desigualdades (Almeida y Ramírez, 2010). Por lo general, la discriminación racial se expresa en forma de exclusión y la violencia no se manifiesta de forma abierta, sino que adquiere carácter simbólico (Meertens, 2008).

Enseguida, se analiza el problema de los excluidos sociales afrocolombianos vinculados al aula escolar planteando algunos de los interrogantes y consideraciones que desde la bioética se pueden dar, para llegar a su examen desde el principio de respeto por la persona y principio de justicia, amplio campo que con seguridad aproxima a su mejor comprensión. No se pretende, pues, dar una solución o un grupo de posibles soluciones, solo se busca hacer un Ilamado a la reflexión. La intención inicial de este artículo tampoco comprende realizar un examen exhaustivo de todas las implicaciones socioantropológicas, económicas o políticas. Tal examen es necesario hacerlo, con ello se abrirían nuevos interrogantes, nuevos escenarios y probables soluciones; se requiere un análisis que tenga en cuenta la mirada de la diversidad cultural y que aborde la exclusión social en el aula escolar de los niños y niñas afrocolombianos (Buffa, 2007).

\section{Una experiencia local}

A partir de una experiencia vivida en una institución pública localizada en la ciudad de Neiva (Huila), específicamente en una comuna de grandes conflictos sociales, como pobreza, violencia familiar, inseguridad, desempleo, familias disfuncionales, abandono y maltrato infantil. En esta institución ingresó un estudiante afrocolombiano, a quien Ilamaremos Juan, para efectos de su identificación. El primer día que Juan llegó, en sus ojos se mostraba miedo, temor a ser rechazado. Al inicio del periodo escolar ningún niño compartía con él, en sus horas de descanso se limitaba a comer la merienda que le daba el colegio y a estar cerca de su maestra que era muy simpática con él. La maestra empezó a realizar sensibilizaciones 
para hablar sobre las diferencias individuales de las personas, del valor del respeto; en algunos niños resultó y empezaron a saludarlo. Sin embargo, otros niños no permitían ni que se les acercara.

Muy a pesar de interactuar a través del saludo, Juan era muy resistente a establecer relaciones con sus compañeros; en ocasiones, se mostraba agresivo y buscaba aislarse constantemente del grupo. En consecuencia, Juan presentaba muchos llamados de atención por parte de otros maestros, sin razón justificada delante de sus compañeros.

Con el tiempo, Juan no volvió a la escuela; la maestra buscó a sus padres, pero ellos le dijeron que él no quería volver y que por razón de cambio de residencia a la zona rural se irían. Varias semanas después de este encuentro, se conoció que el niño recorre las calles de la comuna con sus hermanos pequeños, jugando descalzos todo el día, sin la compañía de un adulto, con ropas sucias y con el riesgo de ser arrollados por algún vehículo automotor.

\section{Exclusión social}

El concepto de exclusión social, introducido en las ciencias sociales para indicar los grupos de individuos y poblaciones excluidos del desarrollo, se refiere, en el mundo contemporáneo, a la condición humana de individuos y colectividades pertenecientes a sociedades en creciente ruptura de cohesión social y, consecuentemente, de marginación, también creciente, de personas y grupos sociales. La exclusión social es, por ende, un problema real enfrentado por personas y grupos sociales, que puede ser objeto de acciones políticas concretas de los Estados o de movimientos sociales organizados y con poder, pero es también objeto de conocimiento producido por varios ámbitos de las ciencias humanas y sociales (Schramm, 2008).

Desde el Movimiento Social de Comunidades Negras (1998), la exclusión es no admitir que una persona tiene oportunidades y tiene derechos, porque es diferente racialmente a la mayoría de la población. Además, la entienden también como el grado de intolerancia que tiene la sociedad frente a alguna comunidad que presenta discrepancias en cuanto a los estilos de comportamiento social.

No se puede desconocer que la exclusión social no solamente se da por el racismo, como es el caso que se trae a colación en este escrito, sino que hay otros factores que pueden generar esta situación en la escuela, como la discriminación por género, origen étnico, discapacidad, pobreza, $\mathrm{VIH} / \mathrm{sida}$, conflictos armados, entre otros, que se dan por estereotipos construidos en la sociedad. 


\section{La exclusión social en la educación}

Según Fleury (2003), en Colombia, como en el resto de América Latina, la exclusión escolar se ha dejado de ver como un hecho natural y hace parte de la "nueva cuestión social", un problema que reclama respuestas por parte del Gobierno y la sociedad. Pero, a pesar de la construcción de políticas en pro del respeto por las diferencias individuales de cada persona, todavía se encuentra en algunos estudiantes un pensamiento tradicional familiar donde se sesga el respeto hacia las diferencias del individuo.

Peñaflor y Jara (2009) plantean que la educación puede ser un recurso importante para combatir la discriminación, la exclusión y cimentar las bases de la inclusión, brindando una formación integral al ser humano y dándole los recursos necesarios para vivir como miembro pleno de la sociedad. Logra ese cometido cuando tiene pertinencia cultural, cuando valora la diversidad como fuente y recurso del desarrollo y cuando genera espacios abiertos para el diálogo, la convivencia y la construcción de la equidad.

Con frecuencia se argumenta que la educación ha de ayudar a reducir la pobreza y las desigualdades, promover la movilidad social, y mejorar las condiciones de vida de las personas y de las sociedades. Asimismo, en el ámbito educativo se habla de interculturalidad haciendo referencia a un ideal, a un "deber ser o a un proyecto utópico que busca mejorar las relaciones asimétricas entre las culturas para generar intercambios más dialógicos" (Zabala, Cusihuamán y Cuenca, 2005). Sin embargo, para crear relaciones de equidad entre "las culturas" es necesario reflexionar sobre las condiciones que la hacen inviable en la práctica, revisar las raíces de las desigualdades sociales en el acceso a recursos materiales, simbólicos y de una propuesta para superar estas desigualdades (Hirmas y Blanco, 2008).

Según Hirmas y Blanco (2008), la diversidad cultural se ve representada en América Latina y el Caribe por 150 millones de afrodescendientes, la tercera parte de toda la población de América Latina, Ilegados al continente por la marea esclavista. Noventa millones de afroamericanos viven en la pobreza, cantidad que representa el $40 \%$ del total de personas que viven en la pobreza en la región. En Brasil, Colombia y Venezuela vive el $80 \%$ de los afrodescendientes de Iberoamérica. Sus demandas por políticas públicas se enmarcan en las recomendaciones de la III Conferencia Mundial contra el Racismo, realizada en Durban, África del Sur, en el 2001. Junto con la pluralidad étnica, el reconocimiento de la pluralidad racial como constitutiva de las naciones ha sido un hecho que solo se ha iniciado a finales del siglo xx, como es el caso de la Constitución Política de Colombia de 1991. 
A partir de la Declaración de Derechos Humanos (1948), una serie de convenciones y tratados internacionales han ido sentando las bases de derecho internacional en defensa de la igualdad de derechos de las personas y el combate contra la discriminación por motivos de "raza" color u origen nacional. La Convención Internacional sobre la Eliminación de todas las Formas de Discriminación Racial (1965) reafirma la falsedad, lo moralmente condenable y socialmente injusto y peligroso de cualquier doctrina de superioridad basada en la diferenciación racial. Un hito importante en este sentido lo marcó el Primer Congreso de Cultura Negra de las Américas, en 1977, donde se plantó la valorización de la cultura afroamericana, la relevancia de la realización de estudios e investigaciones sobre los elementos culturales de ascendencia africana, y su incorporación en la esfera de la enseñanza.

A pesar de que la diversidad cultural es el ambiente natural en el que los pueblos se desenvuelven, los modelos de desarrollo comúnmente admitidos han prestado poca atención a esta diversidad cultural. Se requiere, por tanto, un diálogo renovado entre cultura y desarrollo. Desde esta perspectiva, el reto de las naciones es el de proveer no solo condiciones de equidad básicas que permitan ampliar las opciones de elección que se deben ofrecer a todos, respetando los deseos de los pueblos originarios, afroamericanos, migrantes, minorías religiosas y otras, para lograr el desarrollo global integral más allá de los aspectos social, cultural y salud, sino, además, condiciones de educación como las que se exponen en este escrito (Hirmas y Blanco, 2008).

Año tras año, se ha buscado despertar en los estudiantes el respeto por las diferencias y cambiar la mentalidad de las nuevas generaciones en cuanto al flagelo del racismo y estimular la solución negociada de conflictos dentro y fuera del aula, con alcance en la comunidad (Hirmas y Blanco, 2008). Sin embargo, todavía se observan algunos sesgos discriminatorios por la diversidad cultural en el aula de clase, en muchas ocasiones generado en su descendencia familiar, por la influencia del contexto social o por los mismos agentes educativos.

\section{Efectos de la exclusión social en la infancia afrocolombiana}

Algunos de los principales efectos de la exclusión social en el entorno escolar de la infancia afrocolombiana incluyen agresividad, deserción, acoso escolar y problemas psicológicos (Garcés, 2004). Las niñas y niños afrocolombianos(as) que sufren la exclusión social en la escuela pueden presentar desmotivación para asistir a la escuela, deserción escolar, problemas de autonomía, inseguridad, agresividad y miedo a afrontar retos que se le presenten en su diario vivir, estas tres últimas condiciones en ocasiones persisten hasta la edad adulta (Castillo y Caicedo, 2012; Parada, 2005). 
Las diversas limitaciones demeritan las libertades instrumentales fundamentales (oportunidades económicas, libertades políticas, servicios sociales, garantía de transparencia y seguridad protectora), cuya expansión Amartya Sen (2000) considera indispensable para el desarrollo, en el sentido de que los niños y las niñas afrocolombianos puedan realizar las cosas que valoran en la vida y ejercer su agencia y autonomía. La vida saludable es afectada por la exclusión social en los países en vías de desarrollo y particularmente en Latinoamérica y el Caribe en donde no se goza de educación plena ni de las garantías del Estado de bienestar para todos o para la mayoría de sus habitantes.

Así mismo, desde una mirada negativa, el respeto y la tolerancia son los valores que más se ven afectados con la exclusión social por raza, es decir, el (no)respeto hacia la diferencia, enmarcado en tomar decisiones basadas en prejuicios o estereotipos sociales y la (in)tolerancia racial que consiste en desconfiar de personas con características físicas y culturales distintas de las nuestras e, incluso, también en despreciarlas, implica conductas abusivas o discriminatorias contra las personas debido a su imaginaria inferioridad, hasta tal punto de llegar a una "violencia moral" que, según Segato (2013), se denomina el conjunto de mecanismos legitimados por la costumbre para garantizar el mantenimiento de preservación de sistemas de estatus relativos entre los términos de raza, pero que también están dentro del control de la permanencia de jerarquías de otros órdenes, como género, el étnico, el de clase, el regional y nacional.

La exclusión social en el aula puede generar violencia moral, a partir de una agresión emocional, aunque no sea ni consciente ni deliberada por parte de sus pares o docentes. Los niños y niñas afrocolombianos están expuestos a la ridiculización, la coacción moral, la sospecha, la intimidación, la condenación de su descendencia raizal, la desvalorización cotidiana como personas, de su personalidad y sus trazos psicológicos, de su cuerpo, de sus capacidades intelectuales, de su trabajo, de su valor moral. Este tipo de violencia puede muchas veces ocurrir sin ninguna agresión verbal, manifestándose exclusivamente con gestos, actitudes, miradas. La conducta opresiva es perpetrada en general por compañeros de la misma clase, niños y niñas de otros grupos, docente del aula y demás docentes de la institución.

\section{Los antecedentes de exclusión social desde la historia del "afrocolombiano"}

Es importante aclarar y precisar los diferentes términos con los que se hace referencia a este grupo poblacional. Afrocolombiano(a), negro(a), afrocolombianeidad, negritud y negritudes son términos utilizados, en numerosas 
ocasiones, indistintamente, sin tener claridad de cuál es la referencia adecuada según el contexto histórico, jurídico, regional y político; sin tener en cuenta las implicaciones de utilizar uno u otro.

Dentro y fuera de las comunidades negras afrocolombianas se ha generado un amplio debate — que aún no se resuelve- acerca de cuál es el etnónimo correcto para denominarlas. Afrocolombiano(a) ha sido el término con el que se ha pretendido el reconocimiento del valor cultural y étnico de estas comunidades con huellas de africanía; sin embargo, los detractores de este término han señalado que este opera a manera de eufemismo que invisibiliza los imaginarios negativos y las prácticas de discriminación alrededor de las negritudes desde el contexto colonial hasta la actualidad. Por esta razón, algunos movimientos se han autodenominado negritudes, para hacer énfasis en la carga peyorativa y en los imaginarios negativos alrededor del color de piel "negro", que hicieron de esta categoría colonial un dispositivo legitimador de marginación social, que debe reconocerse mientras a su vez se lleva a cabo un proceso de resignificación de lo negro y de los aportes afrocolombianos a la construcción de la nación colombiana (Ministerio de Cultura, 2010).

La situación y problemática de esta población afrocolombiana se caracteriza por la explotación de su fuerza de trabajo en los empleos duros, el despojo de sus tierras, el retraso educativo, la pobreza e inhumanidad en las condiciones de vidas familiares, el racismo en las relaciones con las comunidades mestizas blancas, la discriminación racial en la cotidianidad, la exclusión racial en casi todas partes y la ciudadanía incompleta, ciudadanía "recortada" por la violación de sus derechos humanos (Mosquera, 2001). Las graves consecuencias económicas, sociales, culturales, educativas, políticas y espirituales que provocó la institución de la esclavitud están vivas y activas dentro de la sociedad colombiana unidas a los desequilibrios, inseguridades e injusticias propios del modelo de desarrollo económico y social capitalista promovido por las élites dominantes, sus víctimas siguen siendo las poblaciones afrodescendientes que reivindican la verdad, justicia, reparación y equidad social y comunitaria. Actualmente siguen en esta situación. Las cifras oficiales del Departamento Administrativo Nacional de Estadística (DANE, 2016) revelan que el departamento de Chocó, donde la mayor parte de su población es de raza afrocolombiana, fue el de mayor índice de pobreza monetaria, la canasta de bienes y servicios para suplir sus necesidades básicas es de menos de 114692 cop.

Según el Ministerio de Cultura de Colombia (2010), los indicadores de educación muestran tasas de analfabetismo de la población afrocolombiana superiores en 4,5 puntos porcentuales con respecto al resto de la población del país. El analfabetismo se presenta especialmente en su población rural. Las tasas de inasistencia escolar son superiores con 
respecto a la población total, situación que se agudiza en las edades más tempranas, y en las asociadas con la educación superior. Adicionalmente, la población afrocolombiana mayor de 15 años tiene un promedio de menos años de escolaridad con respecto a la población total, con una mayor incidencia en las mujeres.

\section{Enfoque bioético en la exclusión social del racismo en la escuela}

La exclusión social, en particular, es objeto de estudio de las éticas aplicadas y de la bioética, puede, en retorno, servir para orientar y legitimar las propias políticas sociales, de acuerdo con algún sistema de valores vigente $\mathrm{o}$ - dicho de manera más realista— fruto de algún acuerdo entre agentes de sistemas de valores diferentes y en conflicto (como es el caso en la mayoría de las sociedades democráticas y pluralistas).

Como objeto de estudio, constituye uno de los temas transdisciplinarios más discutidos en ética, en política y en el campo de las ciencias humanas y sociales en general, en el cual se relaciona con otros conceptos, tales como daño, pobreza, marginación, capacidades, funcionamiento, acceso a los servicios de salud, mercado de trabajo, educación, distribución de recursos, calidad de vida, justicia, equidad, solidaridad, responsabilidad, ciudadanía, democracia, protección, libertad negativa, agente y paciente moral (Schramm, 2008).

La exclusión social puede caracterizarse como una forma de carencia referente a varios aspectos de la vida humana: material, psíquica, familiar, social y cultural, con varios grados de impacto sobre el bienestar de personas, poblaciones, naciones y regiones enteras del planeta. Puede referirse, por ejemplo, a la falta de acceso a derechos humanos formales (como el reconocimiento ciudadano en el caso de los "sin papeles") o a los derechos humanos fundamentales como el derecho a la vida, a la alimentación, a la salud, a la habitación, al trabajo, a la protección, a la educación y a la cultura o a la pobreza y a la miseria, al desempleo prolongado, a la falta de cobertura por servicios sanitarios y más carencias que, directa o indirectamente, impiden a las personas y grupos sociales el ejercicio efectivo de su ciudadanía, como la participación en las decisiones económicas y políticas que afectan de manera significativa su calidad de vida.

Hoy la exclusión social atañe a la mayoría de la población mundial y tiende a aumentar tanto en sociedades del mundo "desarrollado" como del "subdesarrollado"; o sea, atañe no solamente a las sociedades así llamadas dependientes (o en desarrollo), sino también a las sociedades posindustriales y posnacionales, como muestran los principales indicadores nacionales e 
internacionales; y pudiendo agravarse en el futuro, debido a nuevos problemas emergentes, como los referentes a la calidad del medio ambiente y a la disponibilidad de recursos (Schramm, 2008).

Desde el punto de vista moral, la exclusión es actualmente un indicio de transformación de la identidad del ciudadano entendido en principio como agente moral y político integrado en paciente moral, desamparado y no integrado (Veca, 2005), a quien faltan las condiciones necesarias para poder ejercer sus capacidades y funcionar en la toma de decisiones sobre la vida personal y en común, como lo menciona Sen (2004). Desde el punto de vista moral y político de Schramm (2008), la exclusión social está vinculada a la cultura de los derechos humanos y se refiere a las varias generaciones de derechos: a los derechos de primera generación (o individuales), a los derechos de segunda generación (o "sociales") y a los derechos de tercera generación (que pueden incluir los "derechos ambientales" y los de las "generaciones futuras").

La bioética cada vez se amplía a diferentes campos, ramas o áreas del conocimiento, ya que inicialmente empieza en el campo de la medicina, en esta reflexión se hace hincapié en el área de educación. Inicialmente, la bioética tiene un discurso incluyente que permite que la problemática del racismo no sea exclusivamente del derecho, como lo señala Escobar Triana (2000), al plantear que no es conveniente solucionar estas dificultades solo con leyes, porque para que estas se respeten y se hagan válidas y sostenibles se requiere privilegiar los acuerdos y los consensos; los problemas deben "comprenderse para intentar su solución por medio de acuerdos y criterios éticos que faciliten el cumplimiento de esas leyes" (Escobar Triana, 2002, p. 14). La presencia de la bioética, como indica Escobar, genera esta exigencia: "Se requiere una reflexión bioética, racional y laica, en la que intervengan los diferentes enfoques sobre la vida y las relaciones humanas que permitan vivir en comunidad" (2002, p. 15).

La bioética como norma de vida en la toma de decisiones, se impone en todo el contexto social para contrarrestar razonablemente los desequilibrios que puedan provenir de la discriminación de razas, de género, religión, condición social, económica o política, etc., es decir, lo que de por sí constituye alto riesgo para la convivencia social.

Esta reflexión bioética se realiza a partir del caso planteado al inicio y dos principios éticos básicos del Informe Belmont (Comisión Nacional para la Protección de los Sujetos Humanos de Investigación Biomédica y del Comportamiento, 1979) que son el "respeto por las personas" y la "justicia", los cuales servirán como justificación para evaluar las acciones o conductas humanas de los diferentes autores involucrados dentro del fenómeno racial en la escuela, y que se plantean a continuación: 


\section{Respeto por las personas}

Este respeto a los niños y niñas afrocolombianos(as), al igual que a las demás personas, se da partir de dos convicciones éticas: primero, que deberán ser tratados como agentes autónomos y, segundo, que, ante alguna situación donde su autonomía se vea disminuida o ausente, tienen derecho a ser protegidos. Así, el principio de respeto a las personas se divide en dos exigencias morales separadas: la exigencia de reconocer autonomía, que es cuando el capaz de realizar por sí mismo aquellas tareas y actividades propias de su edad y de su entorno sociocultural; $y$ la exigencia de proteger a aquellos con autonomía disminuida o ausente, que es cuando no se tiene la capacidad de tomar decisiones y actuar acorde a ellas, para lo cual el niño tiene derecho de ser representado y sustituido en sus decisiones por alguien expresa y legalmente habilitado para hacerlo.

Respetar la autonomía de estos niños o de cualquier persona significa dar valor a las opiniones y elecciones autónomas, al mismo tiempo que se evita obstruir sus acciones, a menos que estas sean claramente en detrimento de otros; en ocasiones, cuando la autonomía no se ha desarrollado en el niño, el padre de familia o acudiente, es el que fomenta unos estereotipos sociales que median las decisiones de las relaciones sociales de sus hijos, dentro de los diferentes entornos. La falta de respeto por las diferencias entre los compañeros de estudio hacia los estudiantes afrocolombianos por su color de piel se reflejan mediante la repudiación de las decisiones de esa persona. "La capacidad para hacer decisiones propias madura en el transcurso de la vida del individuo y algunos individuos pierden esta capacidad total o parcialmente debido a enfermedad, incapacidad mental o circunstancias que limitan su libertad severamente" (Comisión Nacional para la Protección de los Sujetos Humanos de Investigación Biomédica y del Comportamiento, 1979, p. 4).

Dentro del caso de Juan, este principio se ve violado por algunos de sus compañeros, ya que a pesar de sensibilizaciones y contextualizaciones de la importancia del respeto por las diferencias de todas las personas, impartidas por la docente, se siguen presentando el prejuicio y las violencias morales por los estereotipos sociales; y por decisiones exógenas a su propia autonomía, actúan de acuerdo con la influencia de conductas y relaciones que han recibido de sus padres o acudientes ante esta población, que se van dando de generación en generación.

Es así como Nussbaum (2008) plantea que las construcciones sociales de la emoción se transmiten mediante el aliento, las acciones y las instrucciones paternales, mucho antes de que la sociedad modele al niño. Se enseña a los niños qué y a quién temer, qué ocasiones de ira son razonables y qué conducta resulta vergonzosa. Asimismo, expone que no habría odio racial 
si no existieran ciertas percepciones de relevancia: que las personas cuyo color de piel sea diferente son amenazantes, peligrosas o malas. Al moldear la forma en que los niños ven los objetos, el ser humano se enfrenta a estas convenciones sociales, que pueden ser discriminatorias o violentas.

Cabe resaltar que esta población que excluye cada vez es menor, debido a la implementación de las políticas y estrategias pedagógicas en defensa de los derechos de humanos y principalmente el de los niños.

A partir del caso, se conciben dos dilemas morales frente a este principio, que es el hecho del abandono del niño del aula por su propia autonomía y por consentimiento de sus padres y la responsabilidad del docente frente a la protección del derecho a la educación de ese menor. ¿Qué decisión tomarías si fueras el docente, el niño o el padre de familia/acudiente?

\section{Justicia}

El principio de justicia consiste en que se le dé a cada persona aquello que es propio o debido. Aristóteles mencionaba que la justicia no era dar a cada quien lo que le corresponde, sino que, más bien, consiste en conocer al ser humano desde sus tres principios diferentes: la razón, el valor y el apetito sensitivo (Ética nicomaquea - Ética Eudemia, Aristóteles, 1988); en la sociedad, la razón son los docentes; el valor, los compañeros de clase, y el apetito sensitivo, los estudiantes excluidos.

Beauchamp y Childress (1999) manifiesta que la justicia exige una repartición igual de beneficios y cargas, que evite discriminaciones e injusticias en las políticas e interacciones en la escuela. La teoría de la justicia, como parte de la filosofía jurídico-política, es una teoría crítica de los sistemas de legitimidad. Toda teoría de la justicia incluye, pues, una teoría de la legitimidad, y son precisamente los valores (Sánchez-Valverde, 2013) los que conectan ambas nociones.

La justicia se relaciona dentro de dos niveles: el social y el individual. Hablar de justicia social establece un principio de la vida en común y, en este sentido, se relaciona con el ámbito del derecho y de la legislación, es aquella que necesita cumplir con las necesidades de los hombres dentro de una sociedad. Por otro lado, la justicia individual busca cumplir con las necesidades del hombre desde lo individual.

En el caso particular, se puede hablar de justicia individual cuando todos los niños o niñas afrocolombianos(as) vinculados al aula escolar, tienen derecho a ser tratados con igualdad a pesar de cada una de las particularidades diferenciales de cada persona. La justicia social va de la mano con la desigualdad social que agrava las condiciones de los más necesitados. La justicia social se refiere de manera general a la inequidad política, y la desigualdad social, a la económica. En cuanto al caso de Juan, 
desde esta perspectiva social, es justo que a cualquier estudiante, a pesar de su diferencia de género, raza, religión, etc., se le reconozcan y se le estimulen sus logros y habilidades, en vez de sus defectos: si ha estudiado mucho y ha hecho un excelente examen, que sea calificado con la puntuación más alta. En cambio, si un compañero ha hecho trampa, debería recibir la calificación más baja. Es apropiado que los niños o niñas que hayan ganado una competencia, por ejemplo, deportiva, reciban el trofeo o medalla correspondiente sin discriminación alguna. Es justo que todos los niños reciban la educación adecuada que merecen, posean tiempo para divertirse, y alguien que los cuide responsable y cariñosamente, sin perjuicios de abandono de los estudios. Por el contrario, resulta totalmente injusto que haya niños que deban dejar de estudiar por situaciones de violencias morales o por despreocupación de padres de familia o, peor aún, por trabajar en condiciones de esclavitud.

\section{Conclusión}

En síntesis, la exclusión educativa es un proceso histórico, cultural y social; una dimensión simbólica que se juega en las instituciones desde lo instituido, lo establecido o fundado, que lleva a la negación de la pluralidad humana. En consecuencia, se debe pensar que transformar esta realidad en la escuela implica reconocer a las niñas y los niños en su singularidad, y posibilitar su propio discurso y acción; también significa la construcción de la pluralidad como condición de toda vida política y no solo la universalización del acceso al sistema educativo, y el reconocimiento de una de las principales diferencias inherentes de los seres humanos, la diversidad, sustento dinámico y cambiante desde el cual el sujeto construye su propia identidad. Lo anterior requiere que la educación a la diversidad implique asegurar el derecho a la identidad propia, respetando a cada uno como es, con sus características biológicas, sociales, culturales y de personalidad, que permiten precisamente la individuación de un sujeto en la sociedad.

Es así que, en concordancia, la exclusión social es objeto relevante de la bioética, considerando que este campo se ocupa tanto del punto de vista descriptivo como del normativo de las prácticas humanas que pueden afectar, de manera sustantiva, a terceros. Dentro de este enfoque, es importante contemplar el principio de responsabilidad que recae sobre la población adulta como principal agente diseñador del futuro, específicamente padres y docentes. Dentro de este contexto, ellos serán los agentes primarios que tendrán la tarea de minimizar los conflictos que se puedan generar dentro del aula escolar por la exclusión social, donde pueda estar afectada la integralidad de las niñas y los niños afrocolombianos, para reconocerlos como sujetos de derechos. 
Desde la reflexión bioética, los principios éticos de justicia y respeto a la persona, dentro del contexto escolar en particular, como ejes preservadores de vida cualificada para todos, llevan a consensos viabilizadores, entre otras cosas, del Estado social de derecho y la justicia con inclusión social educativa plena, es decir, ayudan a la disminución de las desigualdades y discriminaciones, y promueven el ejercicio del respeto a las diferencias individuales de cada uno de los niños y niñas, desde la base de los valores y los derechos humanos, donde se llegue a acuerdos o concesos.

Durante largo tiempo se creyó que lo importante era promover la igualdad en el acceso a la escuela, una cuestión de cantidad, pero hoy se sabe que el ingreso al sistema educativo no es suficiente, pues es necesario asegurar el éxito de cada uno de los estudiantes a partir de sus particularidades individuales.

Los padres de familia y docentes como agentes primarios en la escuela desempeñan un papel activo en la producción y el mantenimiento de condiciones de inclusión social, que puede promover una postura crítica y una profunda acción transformadora que rompa los procesos estructurales y simbólicos implicados en esta.

\section{Referencias}

Almeida, G. y Ramírez, T. (2010). El afrocolombiano en los textos escolares colombianos. Análisis de ilustraciones en tres textos de ciencias sociales de básica primaria. Anuario de Historia Regional y de las Fronteras, 15, 225-244.

Aristóteles. (1993). Ética nicomaquea-Ética Eudemia, trad. y notas Julio Palli Bonet. (Libro 5, pp. 240-266). Madrid: Gredos.

Asamblea General de la onu (1948). Declaración Universal de los Derechos Humanos. Recuperado de http://www.un.org/en/universal-declaration-human-rights/.

Beauchamp, T. y Childress, J. (1999). Principios de ética biomédica. Barcelona: Masson.

Buffa, D. (2007). La situación económica y social de la población negra y sus descendientes en América Latina: un estado de la cuestión. Recuperado de http://www.redaepa.org.ar/jornadas/ixjornadas/resumenes/Se19--Afrodescendientes_Becerra/ponencias/Buffa.pdf.

Buile, N. (2007). ¿Diversidad-inclusión vs. transformación? Agora USB, 7(2), 322-332.

Castillo, E. y Caicedo, J. (2012). Yo no me llamo negrito... Racismo, primera infancia y educación en Bogotá. Ponencia presentada en la Conmemoración Día Mundial Contra el Racismo "Por una educación libre de discriminación: del reconocimiento formal a la garantía real". Bogotá. 
Castillo, S. (2009). La escuela y sus discursos. Los textos escolares como instrumentos de exclusión y segregación. Sociedad y Discurso, 15, 107-124.

Comisión Nacional para la Protección de los Sujetos Humanos de Investigación Biomédica y del Comportamiento (18 de abril de 1979). Informe Belmont. Principios éticos y normas para el desarrollo de las investigaciones que involucran a seres humanos. Recuperado de http://www.conbioetica-mexico.salud.gob.mx/descargas/pdf/normatividad/normatinternacional/10._INTL_Informe_Belmont.pdf.

Departamento Administrativo Nacional de Estadística (DANE). (2016). Pobreza. Recuperado de https://www.dane.gov.co/files/investigaciones/condiciones_vida/pobreza/2016/Choco_Pobreza_2016.pdf.

Escobar Triana, J. (2000). La enseñanza de la bioética general en la construcción de una ética civil. Colección Bios y Ethos 12. Bogotá: Universidad El Bosque.

Escobar Triana, J. (2002). Bioética y conductas sociopáticas. En Bioética y conflicto armado (pp. 14-15). Bogotá: Ediciones Universidad El Bosque.

Fleury, S. (2003). La expansión de la ciudadanía. En VV. AA., Inclusión social y nuevas ciudadanías (pp. 167-193). Bogotá: Pontificia Universidad Javeriana.

Garcés, D. (2004). Aproximación a la situación educativa afrocolombiana. En A. Rojas (comp.), Estudios afrocolombianos aportes para un estado del arte (pp. 147-175). Popayán: Universidad del Cauca.

Hirmas, C. y Blanco, R. (2008). Educación y diversidad cultural: lecciones desde la práctica innovadora en América Latina. Santiago de Chile: Orealc/Unesco.

Meertens, D. (2008). Discriminación racial, desplazamiento y género en las sentencias de la Corte Constitucional. El racismo cotidiano en el banquillo. Universitas Humanística, 66, 83-106.

Ministerio de Cultura (2010). Afrocolombianos, población con huellas de africanía. Recuperado de http://www.mincultura.gov.co/areas/poblaciones/comunidades-negras-afrocolombianas-raizales-y-palenqueras/Documents/Caracterizaci\%C3\%B3n\%20comunidades\%20 negras $\% 20 y \% 20$ afrocolombianas.pdf.

Mosquera, J. (2001). Afrocolombianidad. Boletín del Movimiento Nacional Afrocolombiano Cimarrón. Bogotá, Colombia.

Movimiento Social de Comunidades Negras (1998). El negro en la historia de Colombia (p. 14). Cali, Colombia: Arte litográfico H. V.

Nussbaum, M. (2008). Paisajes del pensamiento: la inteligencia de las emociones. Barcelona: Paidós Ibérica. 
Parada, D. (2005). Percepciones sobre el territorio y su relación en la enseñanza de las ciencias sociales desde una perspectiva de educación intercultural a partir de las expresiones gráfico-plásticas, por niños y niñas de origen afrocolombiano. Escuela Jose María Cordova del municipio de San Onofre, Sucre (tesis de maestría), Universidad de Antioquia, Medellín.

Peñaflor, G. y Jara, L. (2009). Representaciones populares en torno a la discriminación. Lima: Centro de Estudios y Publicaciones, Instituto Bartolomé de Las Casas.

Sánchez-Valverde, C. (2013). De lo legal y lo legítimo. En C. Sánchez-Valverde y A. Montané (coords.), Derechos humanos y educación social (pp. 101-118). Alzira, Valencia: Germania.

Schramm, F. (2008). Exclusión social. En J. C. Tealdi (dir.) Diccionario latinoamericano de bioética (p. 588). Bogotá: Unesco, Red Latinoamericana y del Caribe de Bioética/Universidad Nacional de Colombia.

Segato, R. (2003). Las estructuras elementales de la violencia. Buenos Aires: Universidad Nacional de Quilmes/Prometeo 3010.

Sen, A. (2000). El desarrollo como libertad. Gaceta Ecológica, 55, 14-20.

Sen, A. (2004). Elements of a theory of human rights. Philosophy and Public Affairs, 32(4): 315-356.

Veca, S. (2005). La priorità del male e l'offerta filosofica. Milán: Giangiacomo Feltrinelli Editore.

Zavala, V., Cuenca, R. y Córdova, G. (2005). Hacia la construcción de un proceso educativo intercultural: elementos para el debate. Lima: MED, Dinfocad, Proeduca-GTz. 